University of Nebraska - Lincoln

DigitalCommons@University of Nebraska - Lincoln

Roman L. Hruska U.S. Meat Animal Research

U.S. Department of Agriculture: Agricultural Center

Research Service, Lincoln, Nebraska

2012

\title{
Associations of genetic markers in cattle receiving differing implant protocols
}

\author{
D. A. King \\ USDA-ARS, andy.king@usda.gov
}

S. D. Shackelford

USDA-ARS

T. G. McDaneld

USDA-ARS, tara.mcdaneld@ars.usda.gov

L. A. Kuehn

USDA-ARS, Larry.Kuehn@ars.usda.gov

C. M. Kemp

USDA-ARS

See next page for additional authors

Follow this and additional works at: https://digitalcommons.unl.edu/hruskareports

King, D. A.; Shackelford, S. D.; McDaneld, T. G.; Kuehn, L. A.; Kemp, C. M.; Smith, T. P.L.; Wheeler, T. L.; and Koohmaraie, M., "Associations of genetic markers in cattle receiving differing implant protocols" (2012). Roman L. Hruska U.S. Meat Animal Research Center. 413.

https://digitalcommons.unl.edu/hruskareports/413

This Article is brought to you for free and open access by the U.S. Department of Agriculture: Agricultural Research Service, Lincoln, Nebraska at DigitalCommons@University of Nebraska - Lincoln. It has been accepted for inclusion in Roman L. Hruska U.S. Meat Animal Research Center by an authorized administrator of DigitalCommons@University of Nebraska - Lincoln. 


\section{Authors}

D. A. King, S. D. Shackelford, T. G. McDaneld, L. A. Kuehn, C. M. Kemp, T. P.L. Smith, T. L. Wheeler, and M. Koohmaraie 


\title{
Associations of genetic markers in cattle receiving differing implant protocols ${ }^{1,2}$
}

\author{
D. A. King, ${ }^{3}$ S. D. Shackelford, T. G. McDaneld, L. A. Kuehn, C. M. Kemp, \\ T. P. L. Smith, T. L. Wheeler, and M. Koohmaraie ${ }^{4}$
}

USDA ${ }^{5}$-ARS, Roman L. Hruska US Meat Animal Research Center, Clay Center, NE 68933-0166

\begin{abstract}
The potential interaction of growthpromoting implants and genetic markers previously reported to be associated with growth, carcass traits, and tenderness was evaluated. Two implant protocols were applied to subsets of steers $(\mathrm{n}=383)$ and heifers $(n=65)$ that were also genotyped for 47 SNP reported to be associated with variation in growth, fat thickness, LM area, marbling, or tenderness. The "mild" protocol consisted of a single terminal implant $[16 \mathrm{mg}$ estradiol benzoate (EB), $80 \mathrm{mg}$ trenbalone acetate (TBA) or 8 mg EB, $80 \mathrm{mg}$ TBA given to steers and heifers, respectively]. The "aggressive" protocol consisted of both a growing implant ( $8 \mathrm{mg} \mathrm{EB}, 40 \mathrm{mg}$ TBA) for the lightest half of the animals on the aggressive protocol and 2 successive implants (28 mg EB, $200 \mathrm{mg}$ TBA) given to all animals assigned to the aggressive treatment. Implant protocol had measurable impact on BW and ADG $(P$ $<0.05$ ), with the aggressive protocol increasing these traits before the terminal implant (relative to the mild protocol), whereas the mild protocol increased ADG after the terminal implant so that the final BW and ADG over the experimental period were similar between protocols. Animals on the aggressive protocol had sig-
\end{abstract}

nificantly increased $(P<0.05)$ LM area $\left(1.9 \mathrm{~cm}^{2}\right)$, slice shear force $(1.4 \mathrm{~kg})$, and intact desmin (0.05 units), but decreased $(P<0.05)$ marbling score (49 units) and adjusted fat thickness $(0.1 \mathrm{~cm})$, and yield grade $(0.15$ units). Among both treatments, 8 of 9 growth-related SNP were associated with BW or ADG, and 6 of 17 tenderness-related SNP were associated with slice shear force or intact desmin. Favorable growth alleles generally were associated with increased carcass yield traits but decreased tenderness. Similarly, favorable tenderness genotypes for some markers were associated with decreased BW and ADG. Some interactions of implant protocol and genotype were noted, with some growth SNP alleles increasing the effect of the aggressive protocol. In contrast, putative beneficial effects of favorable tenderness SNP alleles were mitigated by the effects of aggressive implant. These type of antagonisms of management variables and genotypes must be accounted for in marker assisted selection (MAS) programs, and our results suggest that MAS could be used to manage, but likely will not eliminate negative impact of implants on quality.

Key words: beef, carcass merit, implant, growth, single nucleotide polymorphism, tenderness

(C) 2012 American Society of Animal Science. All rights reserved.

This document is a U.S. government work and

is not subject to copyright in the United States.

\footnotetext{
${ }^{1}$ Mention of trade names or commercial products in this publication is solely for the purpose of providing specific information and does not imply recommendation or endorsement by the U.S. Department of Agriculture The authors are grateful to Patty Beska, Peg Ekeren, Kristen Ostdiek, Kathy Mihm, Pat Tammen, and Renee Godtel for their assistance in the execution of this experiment and to Marilyn Bierman for her secretarial assistance.

${ }^{2}$ This project was funded, in part, by The Beef Checkoff

${ }^{3}$ Corresponding author: andy.king@ars.usda.gov

${ }^{4}$ Present address: IEH Laboratories and Consulting Group, Lake Forest Park, WA 98155

${ }^{5}$ USDA is an equal opportunity provider and employer.

Received September 13, 2011.

Accepted January 16, 2012.
}

J. Anim. Sci. 2012.90:2410-2423 doi:10.2527/jas2011-4693

\section{INTRODUCTION}

Genetic markers have been developed to aid in selection for a wide variety of economically important traits. However, markers have generally been developed on controlled resource populations, and little is known about their effectiveness under varying management systems. Moreover, few studies have addressed the effects of selection based on markers developed for target traits on other important, off-target traits.

A genome-wide association analysis (GWAS) has recently identified numerous associations between SNP and postweaning growth, $\mathrm{HCW}$, fat thickness, LM area, 
and postweaning growth, HCW, fat thickness, LM area, and tenderness (Snelling et al., 2010). In addition, several SNP have been previously associated with tenderness, including 2 (CAPN1316 and CAPN14751) located in the $\mu$-calpain gene, and 1 (CAST) in the calpastatin gene (Barendse, 2002; Page et al., 2004; White et al., 2005). These SNP have been validated in Bos taurus and Bos indicus cattle (Casas et al., 2006).

Improved production efficiency provides strong incentive for using anabolic implants to optimize growth of beef cattle, despite the detrimental effects of aggressive implant usage on carcass and meat quality (Morgan, 1997; Montgomery et al., 2001; Dikeman, 2003). Currently, cattle may receive multiple implants to increase growth rate and efficiency as they move through various segments of the industry, which may have cumulative effects on meat quality attributes (Samber et al., 1996; Platter et al., 2003). There is little data currently available to address the question of whether individual genetic markers that are successful in resource populations will have similar predictive merit in the context of cattle raised with the use of aggressive implant strategies.

The present study evaluated the predictive merit of genetic markers in cattle produced under differing management practices, and examined if the favorable alleles of SNP will 1) mitigate the negative effects of implant protocols, and 2) affect other traits of economic importance.

\section{MATERIALS AND METHODS}

The use of animals for this experiment was approved by the U.S. Meat Animal Research Center (USMARC) Animal Care and Use committee.

British $\times$ Continental cross steers $(\mathrm{n}=383)$ and heifers $(n=65)$ were used in this study. To facilitate management (including administration of implants) and harvest schedules that fit the weight and progress of the cattle, steers and heifers were divided into 6 and 2 contemporary groups, respectively. Within a contemporary group, cattle were assigned to either an aggressive or mild implant protocol implant treatments so that sire line, sire (if known), dam line, and weaning weight (WW) were balanced across implant treatments, and implant treatments were represented equally within contemporary groups. The WW and implanting protocol for each of these groups are summarized in Table 1. After consultation with industry experts, 2 finishing period implant protocols were designed to have varying impacts on growth rate, carcass merit, and meat quality. Because WW varied greatly, it was determined that the contemporary groups with the lightest WW would require much longer to reach their finished endpoint. Thus, cattle assigned to the aggressive implant protocol from those groups also were given a growing implant containing $8 \mathrm{mg}$ estradiol and $40 \mathrm{mg}$ trenbalone acetate (TBA; Revalor G, Intervet, Inc., Millsboro, DE) at the initiation of the study. A second implant containing $28 \mathrm{mg}$ estradiol benzoate and 200 mg TBA (Synovex Plus, Fort Dodge Animal Health, Overland Park, KS) was applied approximately $75 \mathrm{~d}$ later.

Table 1. Summary of implanting protocols stratified by BW classes during feeding period

\begin{tabular}{|c|c|c|c|c|c|c|c|c|c|}
\hline $\begin{array}{l}\text { Contemporary } \\
\text { group }\end{array}$ & Gender & Implant protocol & $\begin{array}{c}\text { Weaning } \\
\text { weight }\end{array}$ & Grower implant & $\mathrm{d}$ & Implant & d & Terminal implant & d \\
\hline 1 & Steer & Mild & 229.0 & - & - & None & 108 & Revalor IS $^{1}$ & 111 \\
\hline 1 & Steer & Aggressive & 230.2 & - & - & Synovex Plus ${ }^{2}$ & 108 & Synovex Plus ${ }^{2}$ & 111 \\
\hline 2 & Steer & Mild & 223.2 & - & - & None & 96 & Revalor IS ${ }^{1}$ & 127 \\
\hline 2 & Steer & Aggressive & 224.4 & - & - & Synovex Plus ${ }^{2}$ & 96 & Synovex Plus ${ }^{2}$ & 127 \\
\hline 3 & Steer & Mild & 201.1 & - & - & None & 141 & Revalor IS ${ }^{1}$ & 98 \\
\hline 3 & Steer & Aggressive & 201.0 & - & - & Synovex Plus ${ }^{2}$ & 141 & Synovex Plus ${ }^{2}$ & 98 \\
\hline 4 & Steer & Mild & 187.1 & - & - & None & 168 & Revalor IS ${ }^{1}$ & 93 \\
\hline 4 & Steer & Aggressive & 187.1 & - & - & Synovex Plus ${ }^{2}$ & 168 & Synovex Plus ${ }^{2}$ & 93 \\
\hline 5 & Steer & Mild & 172.1 & None & 71 & None & 108 & Revalor IS ${ }^{1}$ & 92 \\
\hline 5 & Steer & Aggressive & 172.4 & Revalor $\mathrm{G}^{3}$ & 71 & Synovex Plus ${ }^{2}$ & 108 & Synovex Plus ${ }^{2}$ & 92 \\
\hline 6 & Steer & Mild & 144.5 & None & 79 & None & 120 & Revalor IS $^{1}$ & 99 \\
\hline 6 & Steer & Aggressive & 146.2 & Revalor $\mathrm{G}^{3}$ & 79 & Synovex Plus ${ }^{2}$ & 120 & Synovex Plus ${ }^{2}$ & 99 \\
\hline 7 & Heifer & Mild & 186.1 & None & 83 & None & 98 & Revalor $\mathrm{IH}^{4}$ & 94 \\
\hline 7 & Heifer & Aggressive & 190.2 & Revalor $\mathrm{G}^{3}$ & 83 & Synovex Plus ${ }^{2}$ & 98 & Synovex Plus ${ }^{2}$ & 94 \\
\hline 8 & Heifer & Mild & 135.7 & None & 64 & None & 122 & Revalor $\mathrm{IH}^{4}$ & 97 \\
\hline 8 & Heifer & Aggressive & 135.6 & Revalor $\mathrm{G}^{3}$ & 64 & Synovex Plus ${ }^{2}$ & 122 & Synovex Plus ${ }^{2}$ & 97 \\
\hline
\end{tabular}

${ }^{1}$ Revalor IS $=16 \mathrm{mg}$ estradiol, $80 \mathrm{mg}$ trenbalone acetate (Intervet, Inc., Millsboro, DE)

${ }^{2}$ Synovex Plus $=28 \mathrm{mg}$ estradiol benzoate, $200 \mathrm{mg}$ trenbalone acetate (Fort Dodge Animal Health, Overland Park, KS)

${ }^{3}$ Revalor $\mathrm{G}=8 \mathrm{mg}$ estradiol, $40 \mathrm{mg}$ trenbalone acetate (Intervet, Inc.)

${ }^{4}$ Revalor $\mathrm{IH}=8 \mathrm{mg}$ estradiol, $80 \mathrm{mg}$ trenbalone acetate (Intervet, Inc.) 
This was the first implant given to the heavier contemporary groups. A terminal implant (Synovex Plus) was administered approximately $100 \mathrm{~d}$ later (approximately $100 \mathrm{~d}$ before harvest) to both the light and heavy contemporary groups within the aggressive treatment. The days between implants varied somewhat between contemporary groups to allow the animals to reach the proper weight and to facilitate scheduling of cattle processing and harvest (Table 1). Steers assigned to the mild implant protocol received a single $16 \mathrm{mg}$ estradiol, $80 \mathrm{mg}$ TBA implant (Revalor IS), whereas heifers assigned to the mild implant protocol received a single $8 \mathrm{mg}$ estradiol, $80 \mathrm{mg}$ TBA implant (Revalor IH, Intervet, Inc.), which was administered at the same time the terminal implant was administered to cattle assigned to the aggressive protocol. The implant given as part of the mild implant protocol is relatively strong compared with other implants that are available (Montgomery et al., 2001). However, using only a single terminal implant, the lifetime protocol was considered mild, especially when compared with the multiple implants in the aggressive protocol. Within a BW group, cattle from each treatment were co-mingled and managed identically.

Cattle were fed a corn- and corn-silage based diet to a final weight appropriate for their frame size as determined by the investigators and the feedlot manager (Supplemental Table 1; http://jas.fass.org/content/vol90/ issue7/). Cattle were weighed each time they were implanted. Within a contemporary group, cattle were fed in the same pen regardless of implant treatment. This was done to minimize the impact of potential environmental effects on carcass and palatability traits, but also precluded the comparison of intake and feed efficiency across implant treatments. Average daily gain was determined (gain during specified time period divided by the number of days in the period) for the period between the initiation of the trial and application of the terminal implant, the period between administration of the terminal implant and harvest, and over the entire experiment.

\section{Genotyping}

Whole blood samples were collected from each animal at the initiation of the experiment and genomic DNA was extracted using commercial kits (Generation Capture Plate Kits, Qiagen, Inc., Valencia, CA) according to the manufacturer's instructions. However, genomic DNA was extracted from ear notches from animals that were born as a twin or that had originated from the USMARC twinning herd using a salt extraction, because contaminating alleles from the co-twin are detected in blood from twin-born calves (Plante et al., 1992; Schellander et al., 1992).
Samples were genotyped for SNP previously identified to be associated with postweaning growth, fat thickness, LM area marbling score, and tenderness in a population of cross-bred steers described by Snelling et al. (2010). However, in some cases where many highly associated SNP were located in close proximity and appeared to be segregating together, 1 or more of the related SNP were omitted and SNP from other regions of the genome were included as an alternative. Additionally, SNP previously reported to affect tenderness in the CAPN1 (Page et al., 2004; White et al., 2005) and CAST (Berendse, 2002) genes also were included. Design and multiplexing by MassARRAY assay design software (Sequenom Inc., San Diego, CA) was performed on a file containing 56 targeted SNP. A target of a maximum of 34 and a minimum of 21 polymorphisms per multiplex was set for design, with default settings for all other parameters. Assays were performed using iPLEX Gold chemistry on a MassArray genotyping system as recommended by the manufacturer (Sequenom Inc.). Genotypes designated as "conservative" calls by the Genotyper software were accepted as correct. Genotypes with lower confidence (marked "moderate" and "aggressive" in the software) were manually evaluated. The CAPN1_316 and CAST polymorphisms did not perform with the multiplex format and were evaluated as single polymorphism assays by use of a homogeneous mass extension MassARRAY assay as recommended by the manufacturer (Sequenom Inc.). The amplification primers for each assay are given in Supplemental Table 2 (http://jas.fass.org/content/vol90/issue7/). Unsuccessful genotyping assays were repeated once, after which samples without genotypes for a given SNP were removed from analyses involving that SNP. Polymorphisms with minor allele frequencies less than 0.15 were excluded from the final analyses. Additionally, 3 SNP had minor allele frequencies greater than 0.15 , but very few animals were homozygous for the minor allele, and thus, animals homozygous for the rare allele were excluded from the final analysis. The 47 SNP included in the final analysis are characterized in Supplemental Table 2 (http://jas.fass. org/content/vol90/issue7/).

\section{Harvest and Carcass Data Collection}

Cattle were harvested in 5 groups at a commercial processing facility via standard procedures. All animals from a given weight group were harvested on the same day. The first 4 groups were stunned by captive bolt before exsanguination. The final harvest group was harvested according to Glatt Kosher ritual harvest.

All carcasses were chilled for $48 \mathrm{~h}$ before being ribbed at the 12th-13th rib interface. Yield grade factors and marbling scores were determined using the USMARC beef carcass grading system (VBG 2000, E + V Technology 
Table 2. Least-squares means of implant protocol effects on meat tenderness and carcass quality traits

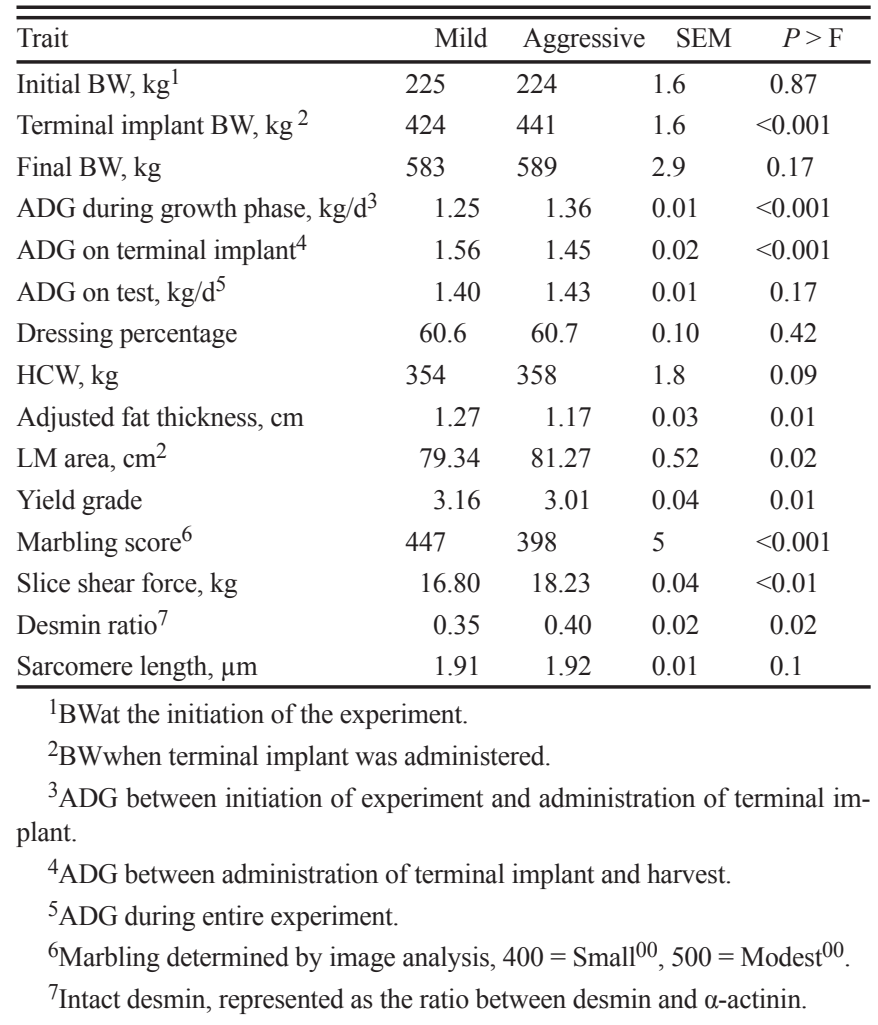

$\mathrm{GmbH}$, Oranienburg, Germany). One 2.54-cm-thick longissimus lumborum steak (13th rib) was removed from the left strip loin of each carcass. Steaks were placed on steel trays and transported, with ice packs, in insulated coolers to the U.S. Meat Animal Research Center. Trays were placed in vacuum bags and aged $\left(1^{\circ} \mathrm{C}\right)$ until $14 \mathrm{~d}$ postmortem. Because steak thickness is critical to beltgrill cookery, the bags were sealed without vacuum during the aging period to avoid compressing the steaks.

\section{Slice Shear Force Determination}

Steaks were allowed to equilibrate in a $5^{\circ} \mathrm{C}$ refrigerator overnight before being cooked on an electric belt grill to an internal temperature of $70^{\circ} \mathrm{C}$ (Wheeler et al., 1998). Slice shear force was determined according to the procedures of Shackelford et al. (1999). The sheared slice was retained from each steak and used for sarcomere length measurement and Western blotting of desmin.

\section{Sarcomere Length and Immunoblotting of Desmin}

Sarcomere length was measured on six $0.5 \times 0.5 \times$ $0.5-\mathrm{cm}$ pieces removed from each slice previously sheared during slice shear force determination. Six fibers were teased from each unfixed sample, and sarcomere length was measured by the laser diffraction method of Cross et al. (1981). Tissue remaining after sarcomere length sam- pling was pulverized in liquid nitrogen. Intact desmin was measured by resolving myofibrillar proteins using SDS PAGE and Western immunoblotting analysis as described by Wheeler et al. (2002) as modified by King et al. (2009). Briefly, the modifications were as follows: Blots were simultaneously probed for desmin (clone D3, developed by D. A. Fischman and obtained from the Developmental Studies Hybridoma Bank maintained by the University of Iowa, Department of Biological Science, Iowa City, IA 52242, under contract N01-HD-7-3263 from the NICHD) and $\alpha$-actinin (monoclonal anti- $\alpha$-actinin clone EA-53; Sigma Chemical Co., St. Louis, MO). Because $\alpha$-actinin does not degrade in postmortem muscle, the intensity of the $\alpha$-actinin band served as an internal standard to evaluate the relative amount of desmin remaining in the sample. Data were expressed as the ratio of the intensity of the desmin band to the intensity of the $\alpha$-actinin band within each sample.

\section{Statistical Analysis}

Data were analyzed using the MIXED procedure (SAS Inst. Inc., Cary, NC). To first establish the effect that implant protocol had on carcass and tenderness traits independent of SNP effects, a model including contemporary group and implant protocol as fixed effects and initial weight as a covariate was used. Animal was considered the experimental unit. A second analysis was then performed where genotype was included to examine the associations of individual markers among treatment protocols. Genotype for each SNP was designated as 0,1 , or 2 with 0 being homozygous for the unfavorable allele (of the originally associated trait), 1 being the heterozygote, and 2 being homozygous for the favorable allele. Animals not successfully genotyped at a given marker were removed from the analysis at that marker. As a result, the number of animals used for the statistical analysis of the effect of each marker and its interaction with implant protocol differed. Markers were fitted individually in separate models along with fixed effects of contemporary group, implant protocol, genotype, and the implant protocol $\times$ genotype interaction. Body weight at the initiation of the experiment (initial BW) was included as a covariate for all traits. Preliminary analyses indicated that the initial weight $x$ implant protocol interaction had no effect on any of the variables and these terms were removed from the model. Least-squares means were generated for both main effects and their interaction. Means for statistically significant effects were separated using the DIFF option and a nominal $\alpha$ of 0.05 because SNP comparisons were considered to be validation of previous results. Bonferoni correction for multiple testing of SNP effects would have required an $\alpha$ of $9.0 \times 10^{-4}$ for statistical significance. Main effects and interactions meeting this criterion are highlighted as well. 


\section{RESULTS AND DISCUSSION}

\section{Implant Effects}

The implants administered to cattle assigned to the mild protocol would be considered a mild combination implant, which is more aggressive than single ingredient estrogenic or androgenic implants (Montgomery et al., 2001). However, we designated this implant protocol as mild in comparison with the aggressive protocol because it used a smaller dose of active ingredients and avoided repeated implants as well as strong implants earlier in the life of the animal. Thus, the impact of the mild protocol on growth and quality traits was expected to be less than the effect of the aggressive protocol (Bartle et al., 1992; Herschler et al., 1995; Morgan, 1997; Dikeman, 2003).

The effects of implant protocol on $\mathrm{BW}$, growth rate, carcass traits, and meat tenderness (using a model with no SNP terms) are shown in Table 3. Body weight was similar between treatments at the beginning of the experiment. However, the implants given to cattle assigned to the aggressive implant protocol during the early part of the feeding period increased $(P<0.05)$ ADG relative to the mild treatment cattle, which were not implanted during this time. As a result, BW at the time the terminal implants were administered was heavier $(P<0.05)$ in the aggressively implanted cattle. After the terminal implants were administered, cattle on the mild implant protocol had greater $(P<0.05)$ ADG until harvest. Thus, contrary to our expectations, final BW and ADG over the entire experiment did not differ between implant protocols. Neither dressing percentage nor $\mathrm{HCW}$ were affected by implant protocol. In partial agreement with the present results, Roeber et al. (2000) found no difference in carcass weight between 7 implant treatments including 1 or 2 applications of implants with differing dosages of estradiol benzoate and TBA. In contrast to the present experiment, Herschler et al. (1995) found that a 1:10 ratio of estradiol-17 $\beta$ : TBA produced greater BW gains than a 1:5 ratio of the same compounds. Furthermore, those investigators found that $28 \mathrm{mg}$ estradiol benzoate and $200 \mathrm{mg}$ TBA (same dosage as the implant used in the aggressive protocol) was near the optimum dosage for rate of BW gain. Scheffler et al. (2003) and Schneider et al. (2007) found that cattle receiving multiple implants had greater $\mathrm{HCW}$ than those receiving a single implant. Furthermore, Platter et al (2003) reported that increasing the number of lifetime implants increased ADG during feeding, final BW and HCW.

The aggressive implant protocols decreased $(P<$ $0.05)$ adjusted fat thickness, and increased $(P<0.05) \mathrm{LM}$ area. Hence, the aggressive implant protocol resulted in a 0.15 unit reduction $(P<0.05)$ in yield grade. Thus, it appears that the aggressive implant protocol both limited fat deposition and increased muscling to a small degree. Marbling score was profoundly affected $(P<0.05)$ by implant protocol, with animals on the mild implant protocol producing carcasses with marbling scores that were 49 units greater than animals given the aggressive protocol. This adverse effect on marbling resulted in a much lower percentage (26 percentage points) of carcasses qualifying for the U.S. Choice grade, which would have a significant impact on carcass value (data not shown). The reduction in marbling score in the present experiment was larger than those reported for similar implant strategies (Morgan, 1997). Both the timing and dosage of implants have been suggested to impact marbling scores. Samber et al. (1996) reported that implant strategies with 2 successive implants that delayed the initial implant until 30 $\mathrm{d}$ on feed did not reduce marbling scores relative to nonimplanted controls, but increased ADG to amounts similar to implant protocols using 3 implants starting on $\mathrm{d} 0$ of the feeding period. Bruns et al. (2005) reported that implanting cattle with a strong combination implant on d 0 of feeding reduced marbling scores relative to nonimplanted controls, but cattle receiving the implant on $\mathrm{d}$ 57 of feeding did not differ from non-implanted controls with regard to marbling score. Roeber et al. (2000) reported that increased dosages of estradiol and TBA and repeated application of these compounds were generally associated with increased LM area and decreased marbling scores. However, in that experiment, the timing of a single application of the implant (d 0 or 59 of feeding) used in the aggressive protocol in the present experiment (Synovex Plus) did not affect marbling score, and both implant times reduced marbling compared with non-implanted controls (Roeber et al., 2003). Herschler et al. (1995) found that a single implant with a dose of estradiol benzoate and TBA, similar to those used for the aggressive implant protocol in the present study, resulted in greater $\mathrm{HCW}$ and lower marbling scores than a single implant similar to the one used in the mild implant protocol in the present study. Bartle et al. (1992) reported linear decreases in marbling score as the dosage of estradiol benzoate and TBA increased. Platter et al. (2003) reported that marbling score decreased as the number of lifetime implants increased.

In the present experiment, the aggressive implant protocol increased $(P<0.05)$ slice shear force values by $1.4 \mathrm{~kg}$ relative to the mild implant protocol. It is possible that the mild protocol had some adverse effect on tenderness relative to non-implanted animals. Increased slice shear force in steaks from cattle treated with the aggressive implant protocol coincided with a greater $(P<0.05)$ amount of desmin remaining after $14 \mathrm{~d}$ of refrigerated storage, indicating that aggressive implant protocols reduced the degradation of cytoskeletal proteins during aging. Schneider et al. (2007) reported that 
Table 3. Least-squares means for effects of SNP previously associated with postweaning BW gain on BW, ADG, and dressing percentage of feedlot cattle on mild or aggressive implant protocols during the feeding period

\begin{tabular}{|c|c|c|c|c|c|c|c|c|c|c|c|c|}
\hline \multirow{3}{*}{ SNP name } & \multirow{3}{*}{ BTA } & \multirow{3}{*}{ Trait $^{1}$} & \multicolumn{10}{|c|}{ SNP allele $^{2}$} \\
\hline & & & \multicolumn{3}{|c|}{0} & \multicolumn{3}{|c|}{1} & \multicolumn{3}{|c|}{2} & \multirow{2}{*}{$P>\mathrm{F}$} \\
\hline & & & Mean & & SEM & Mean & & SEM & Mean & & SEM & \\
\hline ARS-BFGL-NGS-45457 & 6 & TWT & $424^{y}$ & \pm & 2 & $434^{z}$ & \pm & 2 & $440^{\mathrm{z}}$ & \pm & 2 & $4.77 \times 10^{-6}$ \\
\hline ARS-BFGL-NGS-45457 & 6 & FWT & $572^{x}$ & \pm & 3 & $589^{\mathrm{y}}$ & \pm & 3 & $600^{\mathrm{z}}$ & \pm & 4 & $1.92 \times 10^{-7}$ \\
\hline ARS-BFGL-NGS-45457 & 6 & ADGG & $1.25^{\mathrm{x}}$ & \pm & 0.01 & $1.31^{\mathrm{y}}$ & \pm & 0.01 & $1.37^{\mathrm{z}}$ & \pm & 0.02 & $9.54 \times 10^{-7}$ \\
\hline ARS-BFGL-NGS-45457 & 6 & ADGT & $1.44^{\mathrm{y}}$ & \pm & 0.02 & $1.51^{\mathrm{z}}$ & \pm & 0.02 & $1.57^{\mathrm{z}}$ & \pm & 0.03 & $1.66 \times 10^{-3}$ \\
\hline ARS-BFGL-NGS-45457 & 6 & $\mathrm{HCW}$ & $347^{x}$ & \pm & 2 & $358^{y}$ & \pm & 2 & $365^{z}$ & \pm & 2 & $1.21 \times 10^{-7}$ \\
\hline ARS-BFGL-NGS-45457 & 6 & AFT & $1.35^{\mathrm{z}}$ & \pm & 0.03 & $1.22^{\mathrm{y}}$ & \pm & 0.02 & $1.20^{\mathrm{y}}$ & \pm & 0.04 & $2.23 \times 10^{-3}$ \\
\hline ARS-BFGL-NGS-45457 & 6 & LMA & $78.2^{\mathrm{y}}$ & \pm & 0.7 & $81.4^{\mathrm{z}}$ & \pm & 0.5 & $82.9^{\mathrm{z}}$ & \pm & 0.8 & $1.30 \times 10^{-5}$ \\
\hline ARS-BFGL-NGS-45457 & 6 & YG & $3.27^{\mathrm{z}}$ & \pm & 0.06 & $3.07^{\mathrm{y}}$ & \pm & 0.04 & $3.01^{\mathrm{y}}$ & \pm & 0.06 & $2.64 \times 10^{-3}$ \\
\hline Hapmap27083-BTC-041166 & 6 & TWT & $423^{x}$ & \pm & 2 & $432^{y}$ & \pm & 2 & $439^{z}$ & \pm & 2 & $4.77 \times 10^{-6}$ \\
\hline Hapmap27083-BTC-041166 & 6 & ADGA & $1.33^{\mathrm{x}}$ & \pm & 0.01 & $1.40^{\mathrm{y}}$ & \pm & 0.01 & $1.45^{\mathrm{z}}$ & \pm & 0.01 & $1.41 \times 10^{-7}$ \\
\hline Hapmap27083-BTC-041166 & 6 & $\mathrm{HCW}$ & $345^{\mathrm{x}}$ & \pm & 2 & $357^{y}$ & \pm & 2 & $364^{z}$ & \pm & 2 & $2.77 \times 10^{-7}$ \\
\hline Hapmap27083-BTC-041166 & 6 & LMA & $78.4^{\mathrm{x}}$ & \pm & 0.8 & $80.7^{\mathrm{y}}$ & \pm & 0.5 & $82.7^{\mathrm{z}}$ & \pm & 0.7 & $3.56 \times 10^{-4}$ \\
\hline Hapmap23507-BTC-041133 & 6 & TWT & $421^{x}$ & \pm & 3 & $430^{y}$ & \pm & 2 & $436^{\mathrm{z}}$ & \pm & 2 & $3.16 \times 10^{-4}$ \\
\hline Hapmap23507-BTC-041133 & 6 & FWT & $569^{\mathrm{x}}$ & \pm & 4 & $583^{y}$ & \pm & 3 & $597^{\mathrm{z}}$ & \pm & 4 & $7.54 \times 10^{-6}$ \\
\hline Hapmap23507-BTC-041133 & 6 & ADGG & $1.24^{\mathrm{x}}$ & \pm & 0.02 & $1.30^{\mathrm{y}}$ & \pm & 0.01 & $1.36^{\mathrm{Z}}$ & \pm & 0.02 & $2.87 \times 10^{-5}$ \\
\hline Hapmap23507-BTC-041133 & 6 & ADGT & $1.44^{\mathrm{y}}$ & \pm & 0.03 & $1.50^{\mathrm{y}}$ & \pm & 0.02 & $1.57^{\mathrm{z}}$ & \pm & 0.02 & $3.18 \times 10^{-3}$ \\
\hline Hapmap23507-BTC-041133 & 6 & ADGA & $1.33^{\mathrm{x}}$ & \pm & 0.02 & $1.39^{\mathrm{y}}$ & \pm & 0.01 & $1.45^{\mathrm{z}}$ & \pm & 0.01 & $3.89 \times 10^{-7}$ \\
\hline Hapmap23507-BTC-041133 & 6 & $\mathrm{HCW}$ & $345^{\mathrm{x}}$ & \pm & 3 & $354^{y}$ & \pm & 2 & $363^{z}$ & \pm & 2 & $2.20 \times 10^{-6}$ \\
\hline Hapmap33628-BTC-041023 & 6 & ADGG & $1.25^{\mathrm{x}}$ & \pm & 0.01 & $1.32^{\mathrm{y}}$ & \pm & 0.01 & $1.36^{\mathrm{z}}$ & \pm & 0.02 & $4.71 \times 10^{-7}$ \\
\hline Hapmap33628-BTC-041023 & 6 & ADGA & $1.34^{\mathrm{x}}$ & \pm & 0.01 & $1.41^{\mathrm{y}}$ & \pm & 0.01 & $1.45^{\mathrm{z}}$ & \pm & 0.02 & $7.43 \times 10^{-8}$ \\
\hline Hapmap33628-BTC-041023 & 6 & DP & $60.6^{\mathrm{yz}}$ & \pm & 0.1 & $60.9^{\mathrm{z}}$ & \pm & 0.1 & $60.4^{\mathrm{y}}$ & \pm & 0.2 & $3.57 \times 10^{-2}$ \\
\hline Hapmap33628-BTC-041023 & 6 & $\mathrm{HCW}$ & $346^{\mathrm{y}}$ & \pm & 2 & $358^{z}$ & \pm & 2 & $363^{z}$ & \pm & 2 & $2.98 \times 10^{-7}$ \\
\hline Hapmap33628-BTC-041023 & 6 & $\mathrm{AFT}$ & $1.35^{\mathrm{z}}$ & \pm & 0.03 & $1.22^{\mathrm{y}}$ & \pm & 0.03 & $1.19^{y}$ & \pm & 0.04 & $1.81 \times 10^{-2}$ \\
\hline Hapmap33628-BTC-041023 & 6 & LMA & $78.1^{\mathrm{y}}$ & \pm & 0.7 & $81.5^{\mathrm{z}}$ & \pm & 0.6 & $82.8^{\mathrm{z}}$ & \pm & 0.8 & $1.51 \times 10^{-5}$ \\
\hline Hapmap33628-BTC-041023 & 6 & YG & $3.27^{\mathrm{z}}$ & \pm & 0.06 & $3.05^{\mathrm{y}}$ & \pm & 0.05 & $2.99^{\mathrm{x}}$ & \pm & 0.07 & $2.51 \times 10^{-3}$ \\
\hline BTB-01312521 & 6 & LMA & $78.8^{y}$ & \pm & 0.9 & $80.9^{\mathrm{yz}}$ & \pm & 0.6 & $81.7^{\mathrm{z}}$ & \pm & 0.6 & $2.52 \times 10^{-3}$ \\
\hline BTA-21313-no-rs & 6 & LMA & $78.4^{\mathrm{y}}$ & \pm & 1.0 & $80.4^{\mathrm{yz}}$ & \pm & 0.6 & $81.4^{\mathrm{z}}$ & \pm & 0.6 & $3.28 \times 10^{-2}$ \\
\hline BTB-01312166 & 6 & FWT & $580^{\mathrm{y}}$ & \pm & 4 & $585^{\mathrm{yz}}$ & \pm & 3 & $592^{z}$ & \pm & 3 & $4.09 \times 10^{-2}$ \\
\hline BTB-01312166 & 6 & LMA & $78.9^{\mathrm{y}}$ & \pm & 0.8 & $80.9^{\mathrm{z}}$ & \pm & 0.5 & $81.8^{\mathrm{z}}$ & \pm & 0.6 & $2.52 \times 10^{-2}$ \\
\hline Hapmap42748-BTA-98142 & 6 & $\mathrm{MARB}^{3}$ & $434^{z}$ & \pm & 5 & $418^{y}$ & \pm & 6 & $416^{y}$ & \pm & 12 & $4.56 \times 10^{-2}$ \\
\hline
\end{tabular}

${ }^{\mathrm{x}-\mathrm{Z}}$ Least-squared means, within a row, lacking common superscripts differ $(P<0.05)$.

${ }^{1}$ Trait for which effect was detected; TWT = terminal implant BW, kg; FWT = final BW, $\mathrm{kg} ; \mathrm{ADGG}=\mathrm{ADG}$ before terminal implant, $\mathrm{kg} / \mathrm{d} ; \mathrm{ADGT}=\mathrm{ADG}$ after terminal implant, $\mathrm{kg} / \mathrm{d} ; \mathrm{ADGA}=\mathrm{ADG}$ over entire experiment, $\mathrm{kg} / \mathrm{d} ; \mathrm{DP}=$ dressing percentage; $\mathrm{AFT}=$ adjusted fat thickness, $\mathrm{cm} ; \mathrm{LMA}=\mathrm{LM}$ area, $\mathrm{cm}^{2} ; \mathrm{MARB}=$ Marbling score determined by image analysis, $300=$ Slight $^{00}, 400=$ Small $^{00}, 500=$ Modest $^{00}$; $\mathrm{YG}=$ yield grade; $\mathrm{SSF}=$ slice shear force, $\mathrm{kg}$.

${ }^{2}$ Number of favorable alleles for the SNP (assigned based on the trait for which SNP was previously associated); $0=$ homozygous unfavorable, $1=$ heterozygous, $2=$ homozygous favorable. 
Warner-Bratzler shear force increased linearly with increasing doses of estradiol and TBA received by heifers during the feeding period.

In the present experiment, repeated combination implants did not improve increased rate of gain over the entire experiment, final $\mathrm{BW}$, or $\mathrm{HCW}$ relative to a single smaller dose combination implant, although it is not known what impact the implant protocol may have had on feed efficiency. Relative to the milder implant protocol, the aggressive implant protocol profoundly decreased marbling deposition and reduced tenderness, which would reduce carcass value and decrease customer satisfaction.

\section{SNP Previously Associated with Postweaning Growth}

The SNP studied in the present experiment were selected because of their associations with growth, carcass merit, and palatability traits in the population described by Snelling et al. (2010). Although these SNP were selected based on associations with a particular trait, many also had associations with other traits in that population (T. G. McDoneld, unpublished data). The results of the present study highlight the association of the favorable allele of a polymorphism from our previous results on the originally associated trait, as well as other economically important traits.

Only main effects of individual SNP, and individual SNP $\times$ implant protocol interactions that are statistically significant at the nominal $P<0.05$ amount are discussed. Complete results for the main effects of implant protocol and SNP, as well as their interaction for all of the SNP included in the final analysis, are available in Supplemental Table 3 (http://jas.fass.org/content/vo190/issue 7/). Because the contrasts of SNP associations were considered confirmation of discovery results, nominal $P$-values were used for judgments of statistical significance, and all $\mathrm{SNP}$ main effects and SNP $\times$ implant protocol interactions meeting that criterion are presented. Bonferroni correction of multiple comparisons would have required an $\alpha$ of $9.0 \times 10^{-4}$ for statistical significance in the present study and associations meeting this criterion are also highlighted. Twenty-seven of 98 SNP main effects and 1 of 35 SNP $\times$ implant interaction effects that were significant at the amount of $P<0.05$ met the Bonferroni criterion.

Several regions of BTA 6 were associated with growth traits in the experiment by Snelling et al. (2010). Nine polymorphisms were included in the present experiment because they represented the portions of BTA 6 that were most significantly associated with postweaning growth. Least squares means for SNP main effects and SNP $\times$ implant protocol interactions involving those postweaning BW gain SNP are presented in Tables 3 and 4, respectively. Of the 9 SNP included in the present study, 8 had significant associations with BW or ADG traits in the present ex- periment. Four of these had main effect associations with these traits (Table 3) and 4 were involved in interactions between the SNP and implant protocol (Table 4).

Four of the SNP previously associated with growth were associated with increases $\left(P<9.0 \times 10^{-4}\right)$ in most of BW and growth rate traits evaluated in this study (Table 3 ). These 4 postweaning BW gain polymorphisms are located within a 225,000 base pair region on BTA 6 , and, thus, may be describing the same causative variation. Moreover, these SNP are associated with increased $(P<$ $\left.9.0 \times 10^{-4}\right) \mathrm{LM}$ area, and 3 of them are associated with decreased $(P<0.05)$ adjusted fat thickness and numerical yield grades (indicating increased carcass yield). All 4 of these SNP were associated with fat thickness and LM area in our previous experiment (T. G. McDoneld, unpublished data). One of these polymorphisms had main effect associations with slice shear force (Table 3), while implant protocol affected the associations of 3 of these SNP with slice shear force (Table 4). When cattle were aggressively implanted, these SNP were not associated with slice shear force; however, the favorable allele for growth for these SNP was associated with increased $(P<$ $0.05)$ slice shear force of steaks from carcasses of animals that were treated with the mild implant protocol. Thus, it appears that the increase in growth associated with these SNP and the aggressive implant protocol both produce toughening effects that are not additive.

Three other SNP, located within 112,000 base pairs of one another on BTA 6 , had main-effect associations with LM area (Table 3). These SNP also were involved in SNP $\times$ implant protocol interactions affecting terminal implant BW and ADG before application of the terminal implant (Table 4). These SNP were not associated with these traits in cattle that had not been implanted (mild implant protocol), but were associated with increased $(P<0.05) \mathrm{BW}$ and rate of BW gain in animals that had been aggressively implanted. From these results, it appears that the SNP for growth identified by Snelling et al. (2010) were predictive in an independent population and selecting for the favorable alleles for these SNP also would result in increased carcass mass and muscling, but may reduce tenderness in addition to increasing growth. However, the associations of some of these SNP were not evident in the absence of aggressive growth promotants.

\section{SNP Previously Associated with Carcass Traits}

Least-squares main effect and interaction means involving SNP that were previously associated with carcass traits are presented in Tables 5 and 6, respectively. In contrast to the growth SNP, of the 7 SNP selected for inclusion in this study because they previously were associated with fat thickness, only 1 was associated with adjusted fat thickness in the present study. This SNP association with 


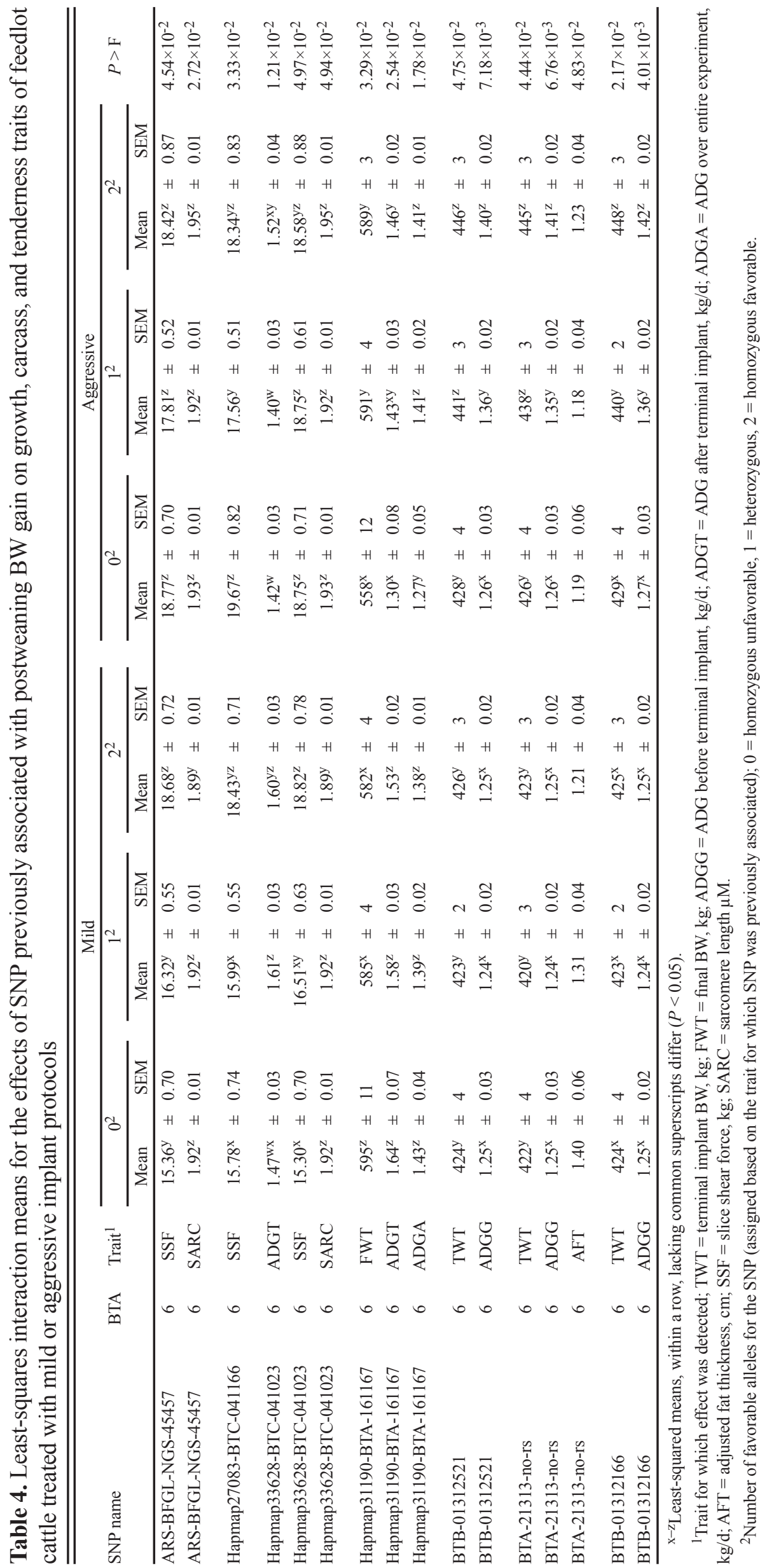


Table 5. Least-squares means for effects of SNP previously associated with fat thickness, LM area, and marbling score on growth, carcass, and meat tenderness traits of feedlot cattle on mild or aggressive implant protocols during the feeding period

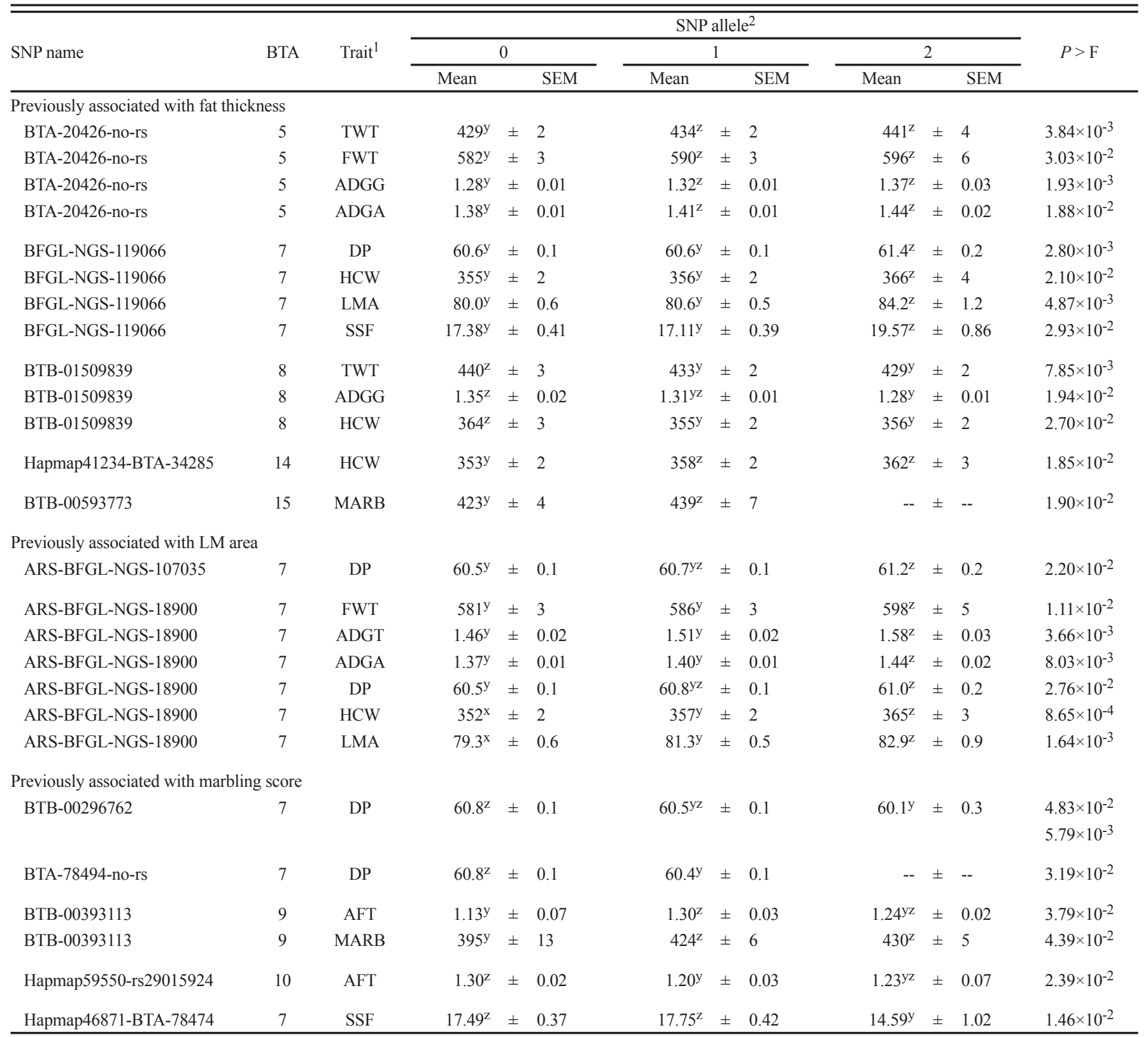

${ }^{\mathrm{x}-\mathrm{Z}}$ Least-squared means, within a row, lacking common superscripts $\operatorname{differ}(P<0.05)$.

${ }^{1}$ Trait for which effect was detected; TWT = terminal implant BW, kg; FWT = final BW, kg; ADGG = ADG before terminal implant, $\mathrm{kg} / \mathrm{d} ; \mathrm{ADGT}=\mathrm{ADG}$ after terminal implant, $\mathrm{kg} / \mathrm{d} ; \mathrm{ADGA}=\mathrm{ADG}$ over entire experiment, $\mathrm{kg} / \mathrm{d} ; \mathrm{DP}=$ dressing percentage; $\mathrm{AFT}=$ adjusted fat thickness, $\mathrm{cm} ; \mathrm{LMA}=\mathrm{LM}$ area, $\mathrm{cm}^{2} ; \mathrm{MARB}=$ Marbling score determined by image analysis, $300=$ Slight $^{00}, 400=$ Small $^{00}, 500=$ Modest $^{00} ; \mathrm{YG}=$ yield grade; $\mathrm{SSF}=$ slice shear force, $\mathrm{kg}$.

${ }^{2}$ Number of favorable alleles for the SNP (assigned based on the trait for which SNP was previously associated); $0=$ homozygous unfavorable, $1=$ heterozygous, $2=$ homozygous favorable.

adjusted fat thickness was involved with an interaction with implant protocol in the present study (Table 6). When given the aggressive implant protocol, adjusted fat thickness did not differ $(P>0.05)$ among the genotypes for this SNP. However, when given the mild implant protocol, animals that were heterozygous for this SNP were associated $(P<0.05)$ with less subcutaneous fat than those that were homozygous for the unfavorable allele. Two of the fat thickness SNP affected growth traits in the present experiment (Table 5) although 1 was associated with increased growth, whereas the other was associated with decreased ADG before administration of the terminal implant.

Five SNP previously associated with LM area were included in the present study. Of these, only 1 was 


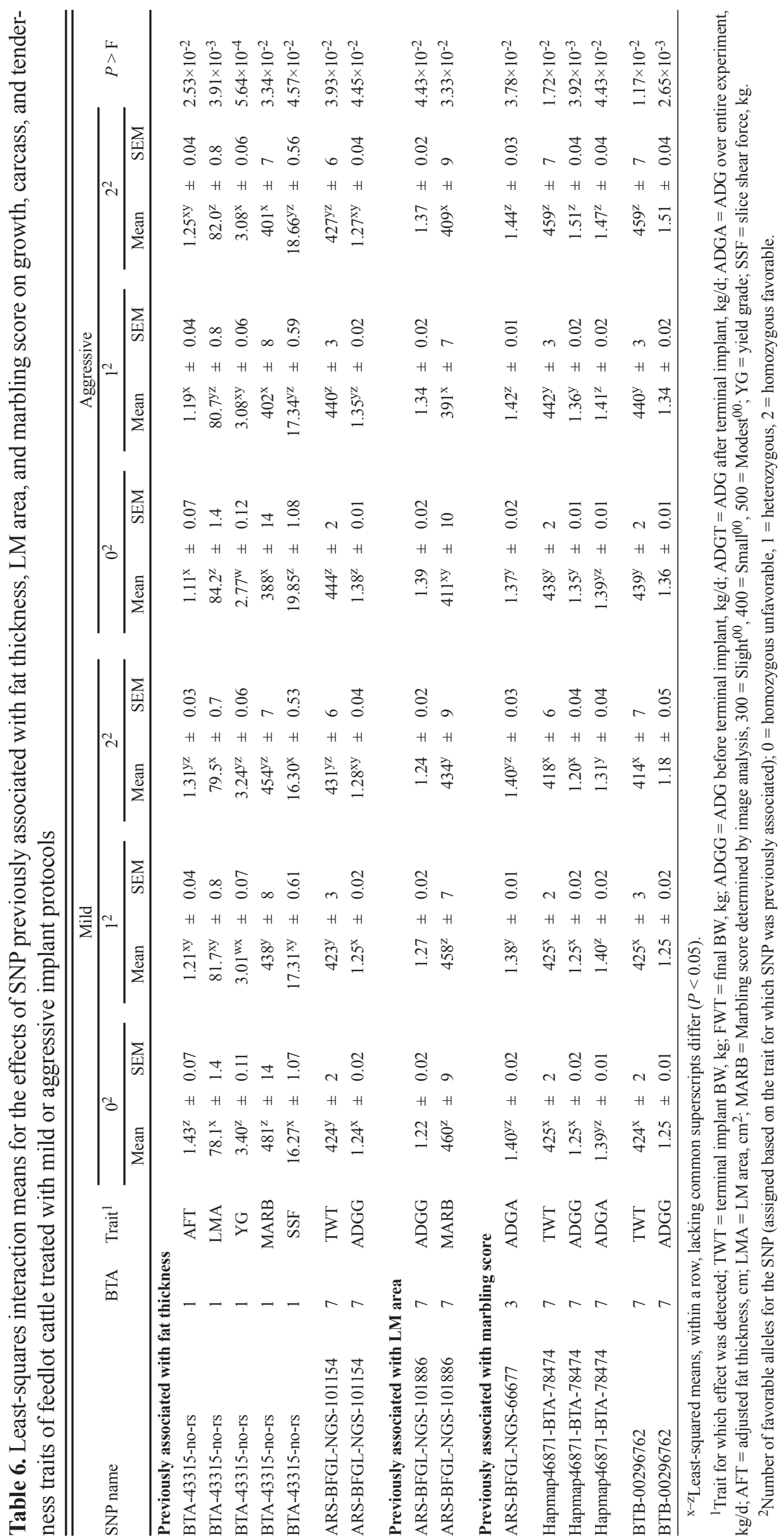


Table 7. Least-squares means for effects of SNP previously associated with tenderness on growth traits of feedlot cattle on mild or aggressive implant protocols during the feeding period

\begin{tabular}{|c|c|c|c|c|c|c|c|c|c|c|c|c|}
\hline \multirow{3}{*}{ SNP name } & \multirow{3}{*}{ BTA } & \multirow{3}{*}{ Trait $^{1}$} & \multicolumn{9}{|c|}{${\text { SNP } \text { allele }^{2}}^{2}$} & \multirow{3}{*}{$P>\mathrm{F}$} \\
\hline & & & \multicolumn{3}{|c|}{0} & \multicolumn{3}{|c|}{1} & \multicolumn{3}{|c|}{2} & \\
\hline & & & Mean & & SEM & Mean & & SEM & Mean & & SEM & \\
\hline Hapmap47381-BTA-70830 & 4 & $\mathrm{DP}$ & $60.6^{\mathrm{yz}}$ & \pm & 0.1 & $60.9^{z}$ & \pm & 0.1 & $60.0^{\mathrm{y}}$ & \pm & 0.3 & $2.07 \times 10^{-2}$ \\
\hline Hapmap47381-BTA-70830 & 4 & YG & $3.10^{\mathrm{y}}$ & \pm & 0.04 & $3.09^{y}$ & \pm & 0.05 & $3.44^{\mathrm{z}}$ & \pm & 0.14 & $4.54 \times 10^{-2}$ \\
\hline ARS-BFGL-NGS-19947 & 6 & TWT & $425^{\mathrm{y}}$ & \pm & 3 & $436^{\mathrm{Z}}$ & \pm & 2 & $431^{y}$ & \pm & 2 & $3.81 \times 10^{-3}$ \\
\hline ARS-BFGL-NGS-19947 & 6 & ADGG & $1.24^{\mathrm{x}}$ & \pm & 0.02 & $1.33^{\mathrm{z}}$ & \pm & 0.01 & $1.30^{\mathrm{y}}$ & \pm & 0.01 & $5.05 \times 10^{-4}$ \\
\hline BTA-87989-no-rs & 6 & ADGG & $1.34^{\mathrm{z}}$ & \pm & 0.01 & $1.29^{\mathrm{y}}$ & \pm & 0.01 & $1.29^{\mathrm{y}}$ & \pm & 0.02 & $4.20 \times 10^{-2}$ \\
\hline BTA-87989-no-rs & 6 & ADGA & $1.43^{z}$ & \pm & 0.01 & $1.39^{\mathrm{y}}$ & \pm & 0.01 & $1.38^{\mathrm{y}}$ & \pm & 0.01 & $3.64 \times 10^{-2}$ \\
\hline BTA-87989-no-rs & 6 & $\mathrm{HCW}$ & $361^{z}$ & \pm & 2 & $355^{\mathrm{y}}$ & \pm & 2 & $356^{\mathrm{yz}}$ & \pm & 2 & $4.20 \times 10^{-2}$ \\
\hline BTB-01904349 & 6 & ADGA & $1.42^{z}$ & \pm & 0.01 & $1.39 \mathrm{yz}$ & \pm & 0.01 & $1.38^{\mathrm{y}}$ & \pm & 0.01 & $3.89 \times 10^{-2}$ \\
\hline BTB-01788557 & 6 & ADGT & $1.50^{\mathrm{y}}$ & \pm & 0.02 & $1.48^{\mathrm{y}}$ & \pm & 0.02 & $1.58^{\mathrm{z}}$ & \pm & 0.03 & $1.74 \times 10^{-2}$ \\
\hline BTB-01788557 & 6 & ADGA & $1.39^{\mathrm{y}}$ & \pm & 0.01 & $1.39^{\mathrm{y}}$ & \pm & 0.01 & $1.43^{z}$ & \pm & 0.02 & $4.97 \times 10^{-2}$ \\
\hline BTB-01788557 & 6 & DP & $61.0^{\mathrm{z}}$ & \pm & 0.1 & $60.5^{y}$ & \pm & 0.1 & $60.6^{\mathrm{y}}$ & \pm & 0.2 & $3.18 \times 10^{-2}$ \\
\hline BTB-01788557 & 6 & $\mathrm{HCW}$ & $358^{\mathrm{yz}}$ & \pm & 2 & $354^{y}$ & \pm & 2 & $362^{z}$ & \pm & 3 & $3.17 \times 10^{-2}$ \\
\hline CAST & 7 & SSF & $19.95^{z}$ & \pm & 1.80 & $18.21^{\mathrm{yz}}$ & \pm & 0.58 & $16.89^{y}$ & \pm & 0.37 & $5.11 \times 10^{-2}$ \\
\hline Hapmap48501-BTA-87072 & 7 & AFT & $1.27^{\mathrm{yz}}$ & \pm & 0.06 & $1.20^{\mathrm{y}}$ & \pm & 0.03 & $1.29^{\mathrm{z}}$ & \pm & 0.02 & $2.42 \times 10^{-2}$ \\
\hline ARS-BFGL-NGS-43901 & 7 & SSF & $19.43^{\mathrm{z}}$ & \pm & 0.78 & $17.96^{\mathrm{z}}$ & \pm & 0.41 & $16.42^{y}$ & \pm & 0.40 & $5.91 \times 10^{-4}$ \\
\hline ARS-BFGL-NGS-43901 & 7 & DES & $0.48^{\mathrm{z}}$ & \pm & 0.03 & $0.39^{\mathrm{y}}$ & \pm & 0.02 & $0.38^{\mathrm{y}}$ & \pm & 0.02 & $2.68 \times 10^{-2}$ \\
\hline CAPN1_316 & 29 & DES & $0.43^{\mathrm{z}}$ & \pm & 0.02 & $0.35^{\mathrm{y}}$ & \pm & 0.02 & $0.25^{\mathrm{y}}$ & \pm & 0.07 & $1.30 \times 10^{-3}$ \\
\hline CAPN1_4751 & 29 & FWT & $595^{z}$ & \pm & 4 & $590^{z}$ & \pm & 3 & $578^{y}$ & \pm & 3 & $1.87 \times 10^{-3}$ \\
\hline CAPN1_4751 & 29 & ADGT & $1.57^{\mathrm{z}}$ & \pm & 0.03 & $1.52^{\mathrm{z}}$ & \pm & 0.02 & $1.46^{\mathrm{y}}$ & \pm & 0.02 & $4.36 \times 10^{-3}$ \\
\hline CAPN1_4751 & 29 & ADGA & $1.43^{z}$ & \pm & 0.02 & $1.41^{\mathrm{z}}$ & \pm & 0.01 & $1.36^{\mathrm{y}}$ & \pm & 0.01 & $6.37 \times 10^{-4}$ \\
\hline CAPN1_4751 & 29 & $\mathrm{HCW}$ & 362 & $\mathrm{z}_{ \pm}$ & 3 & 359 & $\mathrm{z}_{ \pm}$ & 2 & & $\mathrm{y}_{ \pm}$ & 2 & $6.36 \times 10^{-3}$ \\
\hline
\end{tabular}

${ }^{\mathrm{x}-\mathrm{Z}}$ Least-squared means, within a row, lacking common superscripts $\operatorname{differ}(P<0.05)$.

${ }^{1}$ Trait for which effect was detected; TWT = terminal implant BW, kg; FWT = final BW, kg; ADGG = ADG before terminal implant, $\mathrm{kg} / \mathrm{d} ; \mathrm{ADGT}=\mathrm{ADG}$ after terminal implant, $\mathrm{kg} / \mathrm{d} ; \mathrm{ADGA}=\mathrm{ADG}$ over entire experiment, $\mathrm{kg} / \mathrm{d} ; \mathrm{DP}=$ dressing percentage; $\mathrm{AFT}=$ adjusted fat thickness, $\mathrm{cm} ; \mathrm{YG}=$ yield grade; $\mathrm{SSF}=$ slice shear force, $\mathrm{kg}$; DES = Intact desmin present at $14 \mathrm{~d}$ postmortem (represented by ratio of desmin to $\alpha$-actinin in Western blot).

${ }^{2}$ Number of favorable alleles for the SNP (assigned based on the trait for which SNP was previously associated); $0=$ homozygous unfavorable, $1=$ heterozygous, $2=$ homozygous favorable.

associated with increased $(P<0.05)$ LM area in the present study (Table 5). The alleles of this SNP associated with larger LM area were also associated $(P<0.05)$ with increased final BW, ADG after the terminal implant and over the entire experiment, dressing percentage, and HCW. Of these associations, only the association with $\mathrm{HCW}$ exceeded the level required by Bonferroni correc- tion. Only 1 of the 9 SNP included in the present study, because they were previously associated with increased marbling score, was associated $(P<0.05)$ with this trait in the present study. The allele of this SNP associated with increased marbling score also was associated $(P<0.05)$ with increased fat thickness. Implant protocol interacted with the associations of 3 marbling score SNP with growth 
traits, particularly those recorded early in the feeding period (Table 6). The general trends of these interactions indicate that polymorphisms had little effect in animals treated with the mild implant protocol, but the favorable alleles for marbling were associated with increased BW or rate of gain, primarily before application of the terminal implant, in animals treated with the aggressive implant protocol. The animals on the mild implant protocol had not received an implant at this point. Thus, it appears that these SNP had a synergistic effect with the aggressive implant protocol, but no effect in mild-implanted cattle. Few of the SNP included in this experiment because of previous associations with carcass traits had any association with those traits in the present experiment, but impacted other traits, especially growth and BW.

\section{SNP Previously Associated with Tenderness}

Least-squares main effect and interaction means for the tenderness SNP that were associated with the traits evaluated in the present study are presented in Tables 7 and 8, respectively. Seventeen SNP were included in the present study because they had previously been associated with tenderness. Six of these SNP were nominally associated with slice shear force or degradation of the cytoskeletal protein desmin, and 1 of those was associated with slice shear force at the Bonferroni amount. Five SNP located on BTA 6 were associated with BW, HCW, and rate of gain. In three of those SNP, the favorable allele for tenderness was associated $(P<0.05)$ with decreased $(P<$ $0.05) \mathrm{HCW}$. Four of the BTA $6 \mathrm{SNP}$ were associated $(P<$ $0.05)$ with $\mathrm{ADG}$ at some point during the feeding period, though their effects differed. One SNP was associated with increased $\left(P<9.0 \times 10^{-4}\right)$ growth before application of the terminal implant, and was also associated with decreased $(P<0.05)$ intact desmin in longissimus steaks at $14 \mathrm{~d}$ postmortem.

Four of the tenderness SNP included in the present study are located on BTA 7 in close proximity to the CAST gene which codes for calpastatin, inhibitor of the calpains. The CAST SNP was originally reported by Berendse (2002), and has since been confirmed to affect tenderness in both Bos taurus and Bos indicus cattle populations (Casas et al., 2006). In the present experiment, the CAST SNP was associated with decreased $(P<0.05)$ slice shear force of longissimus steaks that had been aged for $14 \mathrm{~d}$ from these cattle. Moreover, 2 other SNP located in close proximity to the calpastatin gene also were associated with decreased $(P<0.05)$ slice shear force. The favorable allele of one SNP also was associated with less $(P<0.05)$ intact desmin.

Two commercially available SNP that are associated with the gene responsible for $\mu$-calpain, the enzyme responsible for postmortem proteolysis, and, consequently, 
tenderization during aging were associated with slice shear force. The favorable allele of CAPN1_316, which was initially reported by Page et al. (2004), was associated with decreased $(P<0.05)$ slice shear force and less $(P<0.05)$ intact desmin in longissimus steaks aged for $14 \mathrm{~d}$. The favorable allele for CAPN1_316 also was associated with greater $(P<0.05)$ numerical yield grades. CAPN1_4751 was originally reported by White et al. (2005) to be associated with tenderness. In the present experiment, the allele of this SNP that is favorable for tenderness was associated with lighter $(P<0.05)$ final BW and HCW. The favorable allele of this SNP also was associated with decreased $(P<0.05)$ ADG after application of the terminal implant and over the entire experiment. Implant protocol interacted with the CAPN1_4751 SNP association with slice shear force (Table 8). On the mild implant protocol, animals homozygous for the unfavorable allele of CAPN1_4751 had slice shear force values similar to those of animals on the aggressive implant protocol. However, animals possessing the favorable allele of this SNP produced steaks with decreased $(P<0.05)$ slice shear force values than those with only the unfavorable allele. Among cattle on the aggressive implant protocol, animals heterozygous for CAPN1_4751 produced steaks with lower $(P<0.05)$ slice shear force values than those that were homozygous for the unfavorable allele, but slice shear force values of steaks from animals homozygous for the favorable allele did not differ from the slice shear force values of steaks derived from animals that were homozygous for the unfavorable allele. Both White et al. (2005) and Casas et al. (2006) found this SNP to be associated with increased tenderness in their studies.

The present experiment supports the previous findings regarding the CAST (Berendse, 2002; Casas et al., 2006), CAPN1 316 (Page et al., 2004; Casas et al,. 2006), and CAPN1_4751 (White et al., 2005; Casas et al., 2006) associations with tenderness. In the present experiment, CAPN1_316 and CAPN1_4751 were associated with decreased carcass yield and decreased growth, BW, and HCW, respectively. Few of the novel tenderness SNP identified in our previous experiments were associated with tenderness in the present experiment. However, several of the SNP that were selected because they were associated with other traits (growth in particular) also were associated with tenderness in the previous experiment (T. G. McDoneld, unpublished data) and were associated with slice shear force in the present experiment as well. Unfortunately, the associations of these SNP on tenderness were generally antagonistic with their effects on their primary trait.

The implant protocols studied in the present experiment created differences in growth, although the net effects of the 2 implant protocols on growth rate and BW over the entire experiment were similar. The aggressive implant protocol did result in some small advantages in carcass composition, as well as considerable reduction in marbling, and a modest reduction in tenderness. Thus, the implant treatments allowed the effects of the SNP to be evaluated under very different production conditions.

Our objectives included evaluating the SNP used in this study for mitigating deleterious effects of the aggressive implant protocol. Most of the SNP associations detected in this experiment were independent of the implant treatment, and the SNP $\times$ implant interactions that were statistically significant at the nominal $P$-value used in this experiment would not be statistically significant at a level corrected for multiple testing, indicating that the SNP associations were additive to implant protocol effects. Thus, selecting for the favorable allele of a given marker should improve the relevant trait, regardless of implanting protocol, which could help manage the deleterious effects of aggressive implant protocols. When SNP associations interacted with implant effects on growth traits, the SNP association generally amplified the growth increase due to the implant protocol. However, when SNP $\times$ implant interactions involved tenderness traits and marbling, the SNP associations generally were not detected in aggressively implanted cattle. Thus, selection using these markers likely would not preferentially mitigate negative effects of aggressive growth promotant strategies on quality, but might be useful in increasing the return from such treatments.

\section{LITERATURE CITED}

Bartle, S. J., R. L. Preston, R. E. Brown, and R. J. Grant. 1992. Trenbalone acetate/estradiol combinations in feedlot steers: Doseresponse and implant carrier effects. J. Anim. Sci. 70:1326-1332.

Barendse, W. J. 2002. DNAmarkers for meat tenderness. International patent application PCT/AU02/00122. International patent publication WO 02/064820 A1.

Bruns, K. W., R. H. Pritchard, and D. L. Boggs. 2005. The effect of stage of growth and implant exposure on performance and carcass composition in steers. J. Anim. Sci. 83:108-116.

Casas, E., S. N. White, T. L. Wheeler, S. D. Shackelford, M. Koohmaraie, D. G. Riley, C. C. Jr. Chase, D. D. Johnson, and T. P. L. Smith. 2006. Effects of calpastatin and $\mu$-calpain markers in beef cattle on tenderness traits. J. Anim. Sci. 84:520-525.

Cross, H. R., R. L. West, and T. R. Dutson. 1981. Comparison of methods for measuring sarcomere length in beef semitendinosus muscle. Meat Sci. 5:261-266.

Dikeman, M. E. 2003. Metabolic modifiers and genetics: Effects on carcass traits and meat quality. International Congress of Meat Science and Technology 49:1-38.

Herschler, R. C., A. W. Olmstead, A. J. Edwards, R. L. Hale, T. Montgomery, R. L. Preston, S. J. Bartle, and J. J. Sheldon. 1995. Production responses to various doses and ratios of estradiol benzoate and trenbalone acetate implants in steers and heifers. J. Anim. Sci. 73:2873-2881.

King, D. A., S. D. Shackelford, T. L. Wheeler, K. D. Pfeiffer, J. M. Mehaffey, M. F. Miller, R. Nickelson, and M. Koohmaraie. 2009. Consumer acceptance and steak cutting yields and of beef top sirloin and knuckle subprimals. Meat Sci. 83:782-787. 
Morgan, J. B. 1997. Implant programs effects on USDA beef carcass quality grade traits and meat tenderness. Pages 147-154 in Proc. Impact of implants on performance and carcass value of beef cattle, Okla. Agric. Exp. Statn,, Stillwater, P-957.

Montgomery, T. H., P. F. Dew, and M. S. Brown. 2001. Optimizing carcass value and the use of anabolic implants in beef cattle. J. Anim. Sci. 79:E296-306.

Page, B. T., E. Casas, R. L. Quaas, R. M. Thallman, T. L. Wheeler, S. D. Shackelford, M. Koohmaraie, S. N. White, G. L. Bennett, J. W. Keele, M. E. Dikeman, and T. P. L. Smith. 2004. Association of markers in the bovine CAPN1 gene with meat tenderness in large crossbred populations that sample influential industry sires. J. Anim. Sci. 82:3474-3481.

Plante, Y., S. M. Schmutz, K. D. Lang, and J. S. Moker. 1992. Detection of leucochimaerism in bovine twins by DNA fingerprinting. Anim. Genet. 23:295-302.

Platter, W. J., J. D. Tatum, K. E. Belk, J. A. Scanga, and G. C. Smith. 2003. Effects of repetitive use of hormonal implants on beef carcass quality, tenderness, and consumer ratings of beef palatability. J. Anim. Sci. 81:984-996.

Roeber, D. L., R. C. Cannell, K. E. Belk, R. K. Miller, J. D. Tatum, and G. C. Smith. 2000. Implant strategies during feeding: Impact on carcass grades and consumer acceptability. J. Anim. Sci. 78:1867-1874.

Samber, J. A., J. D. Tatum, M. I. Wray, W. T. Nichols, J. B. Morgan, and G. C. Smith. 1996. Implant program effects on performance and carcass quality of steer calves finished for 212 days. J. Anim. Sci. 74:1470-1476.
Schellander, K., J. Peli, T. A. Taha, E. Kopp, and B. Mayr. 1992. Diagnosis of bovine freemartinism by the polymerase chain reaction method. Anim. Genet. 23:549-551.

Schneider, B. A., J. D. Tatum, T. E. Engle, and T. C. Bryant. 2007. Effects of heifer finishing implants on beef carcass traits and longissimus tenderness. J. Anim. Sci. 85:2019-2030.

Shackelford, S. D., T. L. Wheeler, and M. Koohmaraie. 1999. Evaluation of slice shear force as an objective method of assessing beef longissimus tenderness. J . Anim. Sci. 77:2693-2699.

Snelling, W. M., M. F. Allan, J. W. Keele, L. A. Kuehn, T. McDaneld, T. P. L. Smith, T. S. Sonstegard, R. M. Thallman, and G. L. Bennett. 2010. Genome-wide association study of growth in crossbred beef cattle. J. Anim Sci. 88:837-848.

Wheeler, T. L., S. D. Shackelford, and M. Koohmaraie. 1998. Cooking and palatability traits of beef longissimus steaks cooked with a belt grill or an open hearth electric broiler. J. Anim. Sci. 76:28052810.

Wheeler, T. L, S. D. Shackelford, and M. Koohmaraie. 2002. Technical note: Sampling methodology for comparing sarcomere length, collagen concentration, and the extent of proteolysis to beef and pork longissimus tenderness. J. Anim. Sci. 80:982-987.

White, S. N., E. Casas, T. L. Wheeler, S. D. Shackelford, M. Koohmaraie, D. G. Riley, C. C. Chase, Jr., D. D. Johnson, J. W. Keele, and T. P. L. Smith. 2005. A new single nucleotide polymorphism in CAPN1 extends the current tenderness marker test to include cattle of Bos indicus, Bos taurus, and crossbred descent. J. Anim. Sci. 83:2001-2008. 